\title{
Improving Orthogonality in Two-Component Biological Signalling Systems using Feedback Control
}

\author{
Harrison Steel, Aivar Sootla, Benjamin Smart, Nicolas Delalez, Antonis Papachristodoulou
}

\begin{abstract}
In natural biological systems, cellular responses to changing growth and environmental conditions are governed by complex signalling and control networks. A common signalling motif is that of the two component signalling systems (TCSS), dozens of which may operate simultaneously in a single cell. When synthetic biologists create new signalling networks in living cells, achieving orthogonality of signal transmission can be difficult. One challenge is overcoming the crosstalk between pathways that arises from off-target interactions between TCSS components. In this paper we analyse a simple signalling network consisting of two parallel TCSS, demonstrating that substantial crosstalk can occur depending on induction levels of each pathway. We then propose and analyse a feedback control architecture that reduces crosstalk by expressing additional substrates depending upon the state of each pathway. We analyse this control architecture's stability, and demonstrate that it facilitates near-orthogonal transmission of signals through each pathway.
\end{abstract}

Index Terms-Biological systems, Control system architecture, Systems biology

\section{INTRODUCTION}

$\mathbf{S}$ YNTHETIC biology is the engineering of biological systems. It exploits the understanding and components found in natural systems to rationally design de novo synthetic systems, or to re-engineer existing ones. A major challenge in synthetic biology is the engineering of systems that implement specific signal processing functions. Cells can process a multitude of external cues, and generate an effective and fast regulation of essential biological processes to successfully adapt to different environments [1]. Prevalent throughout the bacterial kingdom and found in some archaea, plants, and lower eukaryotes are the so-called two component signaling systems (TCSS) for signal transduction [2]. Most bacteria encode dozens, sometimes hundreds, of these signaling proteins. Their modular structure, plasticity, and the great diversity of these systems make them ideal targets for synthetic biological engineering. Potential applications of their signal processing capabilities include signal amplification, logic gates, oscillations, and noise filtering [3], [4], [5], [6]. Recent studies have also demonstrated the potential for cross-kingdom transplantation of TCSS [7], broadening their range of potential applications.

However, when many TCSS pathways operate within a single cell complex interdependencies can arise. This is due to individual enzymes participating in off-target interactions, potentially with several different substrates [8], [9], thereby influencing signals transmitted by other TCSS. In this paper we use crosstalk to refer to such situations, in which the

Authors are with the Department of Engineering Science, University of Oxford, Oxford, OX1 3PJ, UK. e-mail: (\{harrison.steel, antonis\}@eng.ox.ac.uk). H. Steel is supported by the General Sir John Monash Foundation. All authors are supported in part by EPSRC project EP/M002454/1. signalling state of one pathway impacts that of another. In some cases this is a desirable outcome: For example, branched decision-making networks are often found in natural systems [2], crosstalk can pass information between systems [10], [11], and it may even improve signalling robustness [12]. However, this interdependency complicates analysis of signalling systems [13], [14], and may disturb the orthogonality of signal processing in synthetic constructs, making their engineering challenging [15].

Synthetic TCSS for information processing need to be robust, orthogonal, and able to withstand intereference from a host's endogenous processes. To address this engineering challenge in this paper we describe and analyse feedback contol architectures that improve signalling orthogonality by reducing the impact of crosstalk between TCSS. In Section II we describe a simple system with crosstalk, demonstrating how off-target interactions can substantially impact the signalling states of two parallel TCSS. In Section III we investigate feedback control architectures that counteract the influence of crosstalk, finding that they are able to provide each system with orthogonality of signal processing. Stability of the signalling system and the control architectures proposed is then analysed using monotone systems theory in Section IV. In Section V we discuss these results and future work, before the paper is concluded in Section VI.

\section{A Two-Component System with Crosstalk}

In this paper we consider two-component signallng pathways (illustrated in Fig. 1) that are comprised of a Histidine Kinase enzyme $\left(R_{i}\right)$ and a corresponding substrate (responseregulator) upon which it acts $\left(S_{i}\right)$. Each enzyme $R_{i}$ acts upon its substrate as both a kinase (adding phosphate groups) and phosphatase (removing phosphate groups), which controls the proportion of each substrate that is in its phosphorylated state $S_{i}^{P}$. Signals $\left(u_{i}\right)$ enter each pathway via sensory domains attached to each Histidine Kinase. For typical synthetic systems (for example, the synthetic Taz chimera which is used to control the EnvZ/OmpR two-component system [16]) the presence of this input (Aspartate in the case of Taz) decreases the phosphatase activity of EnvZ, whilst its kinase activity remains constant [16]. A range of different inputs can be used in a given pathway, which may have differing effects on the enzyme's behaviour (for example, increasing rather than decreasing its phosphatase activity) [17].

Individual Histidine Kinases have been experimentally observed to phosphorylate a diverse range of substrates, which is not surprising given that they arise from paralogous gene families [2]. This can introduce crosstalk between signalling pathways (dashed lines in Fig. 1), via which the state of 


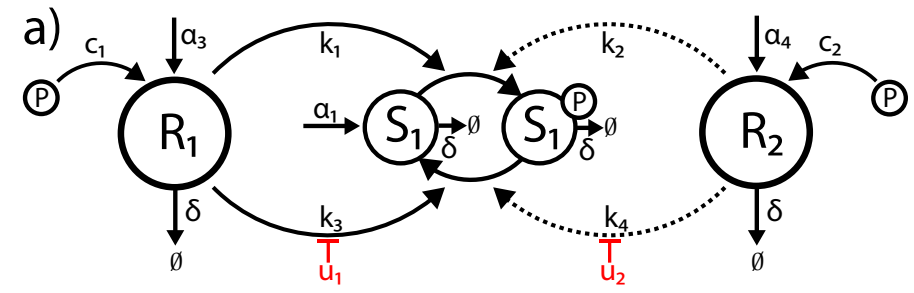

dynamics using eight differential equations:

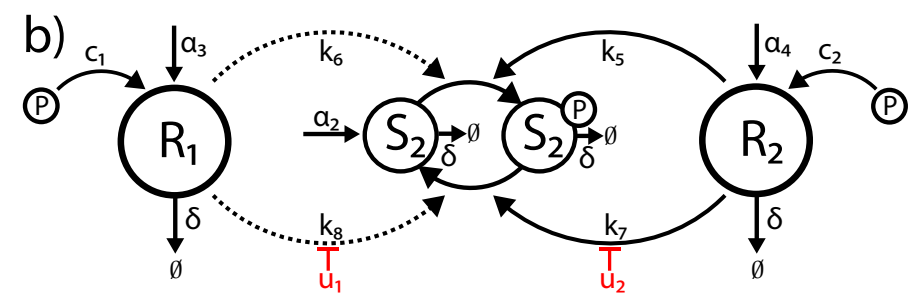

Fig. 1: Two-component system with crosstalk. a) Interactions between substrate $1\left(S_{1}\right)$ and its cognate Histidine Kinase $\left(R_{1}\right)$, as well as crosstalk interactions with a second Histidine Kinase, $R_{2}$. Potentially undesirable crosstalk interactions are indicated by dashed lines. Inducers $\left(u_{i}\right.$, red) decrease the phosphatase activity of each enzyme. b) Similar interaction schematic for substrate 2 .

one pathway can (due to its interactions with non-specific substrates) impact that of another. In many natural systems Histidine Kinases have been shown to have a very strong preference for their corresponding substrate [2], which minimises crosstalk thereby allowing almost orthogonal signal transmission. However, when synthetic signalling pathways are built via mutation or rational engineering of existing systems such orthogonality can be hard to achieve, leading to significant crosstalk.

We build upon the modelling approach of Groban et al. [18] to investigate a simplified system consisting of two generic two-component signalling pathways. This architecture, and the parameter values we choose, is meant to demonstrate the type of crosstalk that might arise in a synthetic signalling pathway, which we then intend to control. This system is illustrated in two parts in Fig. 1, with dashed lines indicating the undesirable non-specific crosstalk interactions. Each input $u_{i}$ serves to reduce the phosphatase activity of its corresponding enzyme, leading to an increased phosphorylation state of its substrate. We initially model our system using eight state variables: $R_{1,2}^{0}$ and $S_{1,2}^{0}$, the concentration of non-phosphorylated Histidine Kinases and substrates respectively, and $R_{1,2}^{T}$ and $S_{1,2}^{T}$, the total concentration of each Histidine Kinase and substrate. These variables are linked via four conservation relations of the form:

$$
\begin{aligned}
& S_{i}^{T}=S_{i}^{P}+S_{i}^{0}, \\
& R_{i}^{T}=R_{i}^{P}+R_{i}^{0},
\end{aligned}
$$

where $S_{i}^{P}$ and $R_{i}^{P}$ are the concentrations of phosphorylated substrate and enzyme respectively, and $i=1,2$. We can describe this system using a chemical reaction network (Supplementary Section I), from which we express the system's

$$
\begin{aligned}
\frac{d S_{1}^{0}}{d t}= & \alpha_{1}-S_{1}^{0}\left(k_{1} R_{1}^{P}+k_{2} R_{2}^{P}\right)+S_{1}^{P}\left(k_{3} R_{1}^{0} f_{1}\left(u_{1}\right)\right. \\
& \left.+k_{4} R_{2}^{0} f_{2}\left(u_{2}\right)\right)-\delta S_{1}^{0}, \\
\frac{d S_{2}^{0}}{d t}= & \alpha_{2}-S_{2}^{0}\left(k_{5} R_{2}^{P}+k_{6} R_{1}^{P}\right)+S_{2}^{P}\left(k_{7} R_{2}^{0} f_{2}\left(u_{2}\right)\right. \\
& \left.+k_{8} R_{1}^{0} f_{1}\left(u_{1}\right)\right)-\delta S_{2}^{0}, \\
\frac{d R_{1}^{0}}{d t}= & \alpha_{3}-c_{1} R_{1}^{0}+R_{1}^{P}\left(k_{1} S_{1}^{0}+k_{6} S_{2}^{0}\right)-\delta R_{1}^{0}, \\
\frac{d R_{2}^{0}}{d t}= & \alpha_{4}-c_{2} R_{2}^{0}+R_{2}^{P}\left(k_{2} S_{1}^{0}+k_{5} S_{2}^{0}\right)-\delta R_{2}^{0}, \\
\frac{d S_{1}^{T}}{d t}= & \alpha_{1}-\delta S_{1}^{T}, \\
\frac{d S_{2}^{T}}{d t}= & \alpha_{2}-\delta S_{2}^{T}, \\
\frac{d R_{1}^{T}}{d t}= & \alpha_{3}-\delta R_{1}^{T}, \\
\frac{d R_{2}^{T}}{d t}= & \alpha_{4}-\delta R_{2}^{T},
\end{aligned}
$$

where $\delta$ is the removal rate of protein due to cell growth, $c_{i}$ is the rate at which each enzyme is phosphorylated, and $\alpha_{i}$ is the production rate (combining transcription and translation) for each protein. Each $f_{i}\left(u_{i}\right)$ is a function of the system's input, which describes how the input signal modulates the phosphatase function of each Histidine Kinase, and is given by:

$$
f_{i}\left(u_{i}\right)=\frac{K_{i}}{u_{i}+K_{i}},
$$

where $K_{i}$ scales the system's saturation in response to inducer $u_{i}$. This functional form reduces each Histidine Kinase's phosphatase activity when an inducer is added, as has been observed experimentally [16]. We can now define what is meant by significant crosstalk in the context of this system, and through that this paper's problem statement:

Problem Statement: Crosstalk from signalling pathway $j$ to $i$ is significant (with significance parametrised by $\epsilon$ ) if at steady-state $M_{i j}\left(u_{i}, u_{j}\right)=\left|\frac{S_{i}^{P}}{S_{i}^{T}}\right|_{u_{i}, u_{j}}-\left.\frac{S_{i}^{P}}{S_{i}^{T}}\right|_{u_{i}, 0} \mid \geq \epsilon$ for any input pair $u_{i}, u_{j}$. Typically $\epsilon$ might be chosen on the order of $\sim 10^{-1}$. We aim to design controllers that allow significant crosstalk to be avoided by reducing $M_{i j}$ for all combinations $f_{i, j}\left(u_{i, j}\right) \in[0.05,1]$ (which corresponds to $0 \leq u_{i}, u_{j} \leq 2$ when $K_{i}=0.1$ ).

Our system's performance will depend on parameter values chosen (outlined in Table I), which may vary substantially depending on the particular two-component system employed [19]. Cognate phosphorylation rates $\left(k_{1,5}\right)$ typically lie in the range $0.1-100 \mu \mathrm{M}^{-1} \mathrm{~s}^{-1}$ [18], [19]. Given that we aim to analyse synthetic signalling networks we selected non-cognate phosphorylation rates $\left(k_{2,6}\right)$ such that each Histidine Kinase shows a 40-fold preference for its own substrate (i.e. $k_{1} / k_{2}=k_{5} / k_{6}=40$ ), though this can be as high as $10^{3}-10^{5}$ for natural systems that evolve strong orthogonality [2], [20]. Cognate de-phosphorylation $\left(k_{3,7}\right)$ has been measured for typical two-component systems in the 


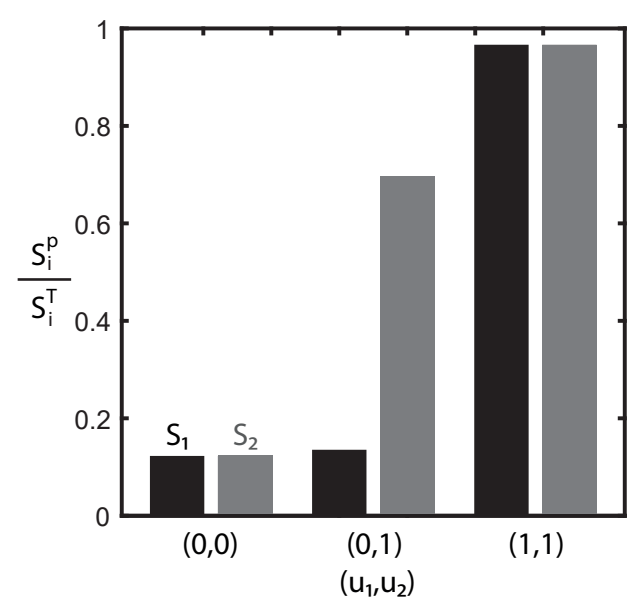

Fig. 2: Strong crosstalk is observed between the actions of $u_{1}$ and $u_{2}$ for the system in Fig. 1. When one input is increased (in this case $u_{2}$ ) its substrate's phosphorylation state $S_{2}^{P} / S_{2}^{T}$ goes from low to high, though minimal change is observed in the state of $S_{1}$. However, when $u_{1}$ is increased crosstalk occurs due to interactions between $R_{1}$ and $S_{2}$, and hence $S_{2}^{P} / S_{2}^{T}$ increases further.

\begin{tabular}{c|c|c} 
Parameter & Value & Unit \\
\hline$k_{1,5}$ & 1.0 & $\mu \mathrm{M}^{-1} \mathrm{~s}^{-1}$ \\
$k_{2,6}$ & $2.5 \times 10^{-2}$ & $\mu \mathrm{M}^{-1} \mathrm{~s}^{-1}$ \\
$k_{3,7}$ & $2.0 \times 10^{-2}$ & $\mu \mathrm{M}^{-1} \mathrm{~s}^{-1}$ \\
$k_{4,8}$ & $1.0 \times 10^{-3}$ & $\mu \mathrm{M}^{-1} \mathrm{~s}^{-1}$ \\
$K_{1,2}$ & $1 \times 10^{-1}$ & $\mu \mathrm{M}$ \\
$c_{1,2}$ & $8.0 \times 10^{-3}$ & $\mathrm{~s}^{-1}$ \\
$\alpha_{1,2}$ & $1.2 \times 10^{-3}$ & $\mu \mathrm{M} \mathrm{s}^{-1}$ \\
$\alpha_{3,4}$ & $1.95 \times 10^{-4}$ & $\mu \mathrm{M} \mathrm{s}^{-1}$ \\
$\alpha_{a, b}$ & $2.4 \times 10^{-4}$ & $\mathrm{~s}^{-1}$ \\
$\delta$ & $3.9 \times 10^{-4}$ & $\mathrm{~s}^{-1}$
\end{tabular}

TABLE I: Nominal parameter values estimated from the literature as discussed in the text. Values are symmetric (i.e. $k_{1}=k_{5}$ ) between the two pathways considered (Fig. 1a,b).

range $2 \times 10^{-3}-5 \times 10^{-2} \mu \mathrm{M}^{-1} \mathrm{~s}^{-1}$ [18], [21], and we set the fold-preference for cognate de-phosphorylation at 20 (i.e. $k_{3} / k_{4}=k_{7} / k_{8}=20$ ) to reflect measured values [18]. Histidine Kinase autophosphorylation rates $\left(c_{1,2}\right)$ in bacteria have been measured in the range $2.0 \times 10^{-3}-1 \times 10^{-1}$ $s^{-1}$ [18], [22] depending on the influence of secondary interactions [22]. $\alpha_{i}$ 's are chosen such that steady-state abundances of each Histidine-Kinase and substrate are 500 and 3000 per cell respectively (past studies have put these values in the ranges $100-2000$ and $3000-6000$ respectively [18], [23]). Finally, $\delta$ corresponds to a cellular doubling time of $\sim 30$ minutes typical of $E$. coli, and $K_{i}$ provides an (essentially arbitrary) scaling for the inducer signal $u_{i}$.

In Fig. 2 we simulate the system in (2). When both inputs are low $\left(u_{1}, u_{2}\right)=(0,0)$ we observe that each substrate $S_{i}$ is mostly de-phosphorylated. However, when inputs are added this phosphorylation state increases for the corresponding substrate: When we set a single input to be non-zero, $\left(u_{1}, u_{2}\right)=(0,1)$, there is minimal crosstalk, with the value

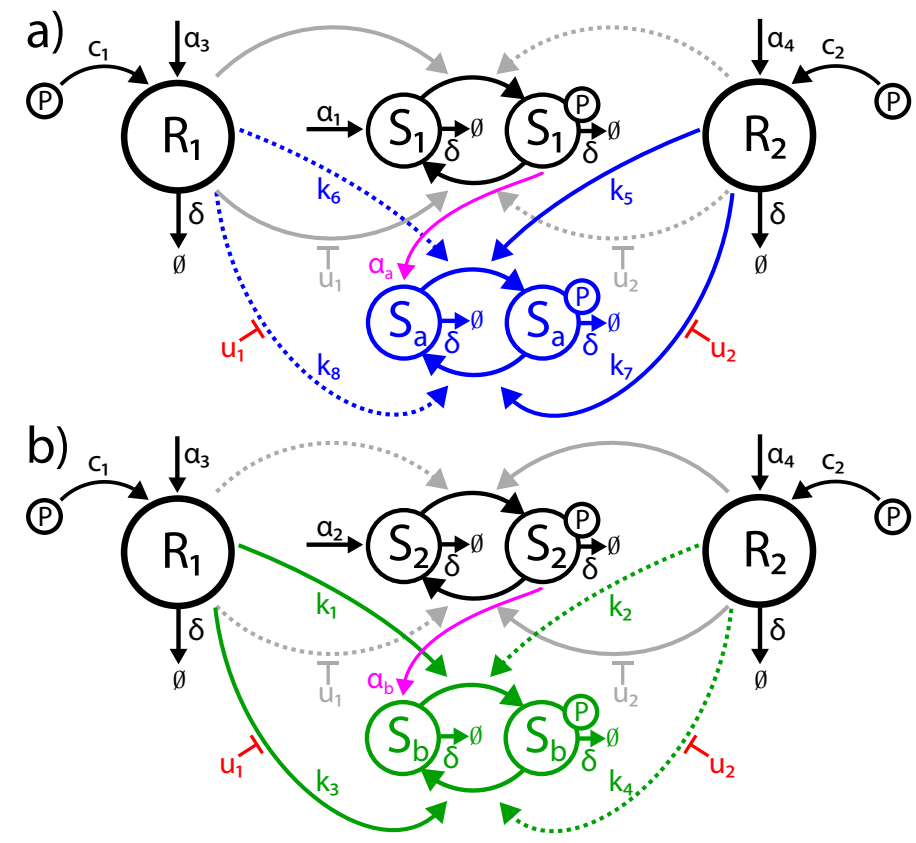

Fig. 3: Two-component system control architectures that mitigate crosstalk. Crosstalk is mitigated by producing alternate substrates, $S_{a}$ and $S_{b}$, which are recognised similarly to $S_{2}$ and $S_{1}$ respectively by their corresponding Histidine Kinases. a) Expression of control species $S_{a}$ (blue), a functionally equivalent substrate to $S_{2}$, decreases the proportion of phosphorylated $R_{2}$ when $S_{1}^{P}$ is high, thereby reducing the rate at which $S_{2}^{P}$ is phosphorylated. b) Similar system, but expressing control species $S_{b}$ (green), a functionally equivalent substrate to $S_{1}$, depending on the concentration of $S_{2}^{P}$.

of $S_{1}^{P}$ remaining similar to that when $u_{2}=0$. However, when we set both $\left(u_{1}, u_{2}\right)=(1,1)$ crosstalk is present, as the changed state of $u_{1}$ has impacted $S_{2}^{P}$ substantially: The value of $S_{1}^{P} / S_{1}^{T}$ which was previously $\sim 0.7$ rises to $\sim 0.95$. In the following sections we demonstrate this crosstalk over a range of input values, and investigate control approaches that reduce its impact.

\section{Sink Control Architecture}

We now implement a feedback control scheme as illustrated in Fig. 3. Here we express new substrates $S_{a}$ and $S_{b}$ that act as "sinks" for phosphate groups from their corresponding Histidine Kinases. This control scheme functions as follows: If $u_{1}$ is increased the proportion of $S_{1}$ in its phosphorylated state will increase, and due to crosstalk so will the proportion of phosphorylated $S_{2}$ (though to a lower extent). In response to the increased $S_{1}^{P}, S_{a}$ (which is preferentially phosphorylated by $R_{2}$ ) will be expressed. This increasing concentration of $S_{a}$ will reduce the phosphorylated proportion of $R_{2}$, leaving less phosphorylated $R_{2}$ to interact with $S_{2}$, thereby reducing the proportion of phosphorylated $S_{2}$ back toward its level prior to $u_{1}$ 's increase. The phosphorylation state of $S_{2}$ will thus (ideally) be made independent of $u_{1}$, and a similar argument applies to the effect of $S_{b}$ on $S_{1}$. 
The sink molecules $S_{a, b}$ could be created by mutating substrates $S_{1,2}$ in such a way that their phosphorylation reactions are maintained, but their down-stream interactions (i.e. promoter recognition by their phosphorylated state) is removed. This control scheme can be implemented in two halves: We can include substrate $S_{a}$ to mitigate the impact of crosstalk between $R_{1}$ and $S_{2}$ (Fig. 3a), or we can include substrate $S_{b}$ to mitigate the impact of crosstalk between $R_{2}$ and $S_{1}$ (Fig. 3b). Both schemes could also be implemented simultaneously to reduce crosstalk in both directions. This control scheme can be described using a set of differential equations of the form:

$$
\begin{aligned}
\frac{d R_{1}^{0}}{d t}= & \alpha_{3}-c_{1} R_{1}^{0}+R_{1}^{P}\left(k_{1} S_{1}^{0}+k_{6} S_{2}^{0}+k_{6} S_{a}^{0}+k_{1} S_{b}^{0}\right) \\
-\delta R_{1}^{0}, & \\
\frac{d R_{2}^{0}}{d t}= & \alpha_{4}-c_{2} R_{2}^{0}+R_{2}^{P}\left(k_{2} S_{1}^{0}+k_{5} S_{2}^{0}+k_{5} S_{a}^{0}+k_{2} S_{b}^{0}\right) \\
-\delta R_{2}^{0} & \\
\frac{d S_{b}^{0}}{d t}= & \alpha_{b} S_{2}^{P}-S_{b}^{0}\left(k_{1} R_{1}^{P}+k_{2} R_{2}^{P}\right)+S_{b}^{P}\left(k_{3} R_{1}^{0} f_{1}\left(u_{1}\right)\right. \\
& \left.+k_{4} R_{2}^{0} f_{2}\left(u_{2}\right)\right)-\delta S_{b}^{0}, \\
\frac{d S_{a}^{0}}{d t}= & \alpha_{a} S_{1}^{P}-S_{a}^{0}\left(k_{5} R_{2}^{P}+k_{6} R_{1}^{P}\right)+S_{a}^{P}\left(k_{7} R_{2}^{0} f_{2}\left(u_{2}\right)\right. \\
& \left.+k_{8} R_{1}^{0} f_{1}\left(u_{1}\right)\right)-\delta S_{a}^{0}, \\
\frac{d S_{a}^{T}}{d t}= & \alpha_{a} S_{1}^{P}-\delta S_{a}^{T}, \\
\frac{d S_{b}^{T}}{d t}= & \alpha_{b} S_{2}^{P}-\delta S_{b}^{T},
\end{aligned}
$$

where $\alpha_{a, b}$ is the feedback strength, which would be implemented by placing the expression of $S_{a, b}$ under the control of a promoter that is regulated by the concentration of phosphorylated substrate $\left(S_{1}^{P}\right.$ and $\left.S_{2}^{P}\right)$ respectively. Here we replace $(2 \mathrm{c}, \mathrm{d})$ with $(4 \mathrm{a}, \mathrm{b})$ respectively, to account for the new substrate's sequestration of phosphate groups from each Histidine Kinase. We also require a new conservation relation:

$$
S_{a, b}^{T}=S_{a, b}^{P}+S_{a, b}^{0} .
$$

To create a one-sided control scheme (i.e. including $S_{a}$ but not $S_{b}$ ) we can set the corresponding creation rate for the other substrate to zero (i.e. $\alpha_{b}=0$ in this case). In Fig. 4 we simulate the two-sided and both one-sided control schemes, demonstrating their ability to reduce crosstalk in the system. In an ideal system the signalling state of one substrate would be independent of the input at the other substrate. We see that this is not the case for the uncontrolled system in Fig. $4 \mathrm{c}$, where the cross-talk $M_{21}$ (and in Fig. S1d the proportion of $S_{2}$ that is phosphorylated) strongly depends on the signal $u_{1}$. Adding the one sided control scheme (including $S_{a}$ to reduce crosstalk between $R_{1}$ and $S_{2}$ ), or the two-sided scheme, mitigates the impact of this crosstalk, reducing the value of $M_{21}$ and $M_{12}$ as $u_{1}$ is varied. In Figs. 4 d-f the dependence of this control scheme on the feedback parameter $\alpha_{a, b}$ (which could be tuned via Ribosome Binding Site adjustment) is illustrated for the two sided control system. Sensitivity to the value of this parameter is discussed in greater detail in Supplementary Section II. Further analysis of our system's parameter sensitivty is provided in Supplementary Section III, in which (2) and (4) are non-dimensionalised to highlight parameter combinations upon which the system's behaviour depends.

\section{ANALYSIS OF THE INTERCONNECTION USING MONOTONE Systems THEORY}

To analyse the stability properties of the crosstalking TCSS and the control approach proposed in Section III we apply results from Monotone Systems Theory [24], outlined in Supplementary Section IV. We refer to the system described in Section III as the closed-loop system. We will call the system open-loop, if in the equations for $S_{a}^{T}, S_{a}^{0}, S_{b}^{T}, S_{b}^{0}$ we replace the protein production rates $\alpha_{a} S_{1}^{P}, \alpha_{b} S_{2}^{P}$ by variables $v_{2}, v_{1}$, respectively.

Theorem 1: Consider the open-loop system, where in the equations for $S_{a}^{T}, S_{a}^{0}, S_{b}^{T}, S_{b}^{0}$ we replace the protein production rates $\alpha_{a} S_{1}^{P}, \alpha_{b} S_{2}^{P}$ by variables $v_{2}, v_{1}$, respectively. Let all the parameters $\left(\alpha_{i}, \alpha_{a}, \alpha_{b}, K_{i}, u_{i}, k_{i}, c_{i}, \delta\right)$ of the system be nonnegative. Then:

a) the open-loop system is forward-invariant with respect to the cone

$$
\begin{gathered}
\mathcal{K}=\left\{R_{i}^{T}, R_{i}^{0}, S_{i}^{T}, S_{i}^{0}, S_{a}^{T}, S_{a}^{0}, S_{b}^{T}, S_{b}^{0} \geq 0\right. \\
\left.S_{i}^{T} \geq S_{i}^{0}, R_{i}^{T} \geq R_{i}^{0}, S_{a}^{T} \geq S_{a}^{0}, S_{b}^{T} \geq S_{b}^{0}\right\}
\end{gathered}
$$

b) Let $\alpha_{1}=B_{1}, \alpha_{2}=B_{2}, v_{1}=0$ and $v_{2}=0$, and consider two trajectories of the open-loop system: The trajectory $\phi_{1}$ with the initial condition

$$
\begin{gathered}
R_{i}^{T}(0)=R_{i}^{0}(0)=\alpha_{i+2} / \delta, S_{i}^{T}(0)=S_{i}^{0}(0)=B_{i} / \delta, \\
S_{a}^{T}(0)=S_{b}^{T}(0)=S_{a}^{0}(0)=S_{b}^{0}(0)=0,
\end{gathered}
$$

where $i=1,2$, and the trajectory $\phi_{2}$ with the initial condition

$$
\begin{gathered}
R_{i}^{T}(0)=\alpha_{i+2} / \delta, S_{i}^{T}(0)=B_{i} / \delta, R_{i}^{0}(0)=S_{i}^{0}(0)=0, \\
S_{a}^{T}(0)=S_{b}^{T}(0)=S_{a}^{0}(0)=S_{b}^{0}(0)=0,
\end{gathered}
$$

where $i=1,2$. If the trajectories $\phi_{1}$ and $\phi_{2}$ converge to the same point, then for every $\alpha_{i}=E_{i}, v_{i}=D_{i}$ with $i=1,2$ such that $E_{i}+D_{i}=B_{i}, E_{i}, D_{i} \geq 0$ the open-loop system has a unique steady-state in $\mathcal{K}$, which lies in the set

$$
\begin{array}{ll}
0 \leq R_{i}^{0} \leq R_{i}^{T}=\alpha_{i+2} / \delta, & 0 \leq S_{i}^{0} \leq S_{i}^{T}=E_{i} / \delta, \\
0 \leq S_{a}^{0} \leq S_{a}^{T}=D_{2} / \delta, & 0 \leq S_{b}^{0} \leq S_{b}^{T}=D_{1} / \delta .
\end{array}
$$

The proof of Theorem 1 is lengthy and can be found in Supplementary Section IV.

Theorem 1 provides an easy algorithm for verifying the existence of the unique steady-state of the open-loop system. The closed-loop analysis, however, is more delicate, and we discuss it in greater detail in Supplementary Section IV-D. As in Theorem 1, we can show that the closed-loop system is invariant with respect to $\mathcal{K}$ and more generally its trajectories are bounded for nonnegative parameter values. In order to perform stability analysis, we fix specific parameter values and 

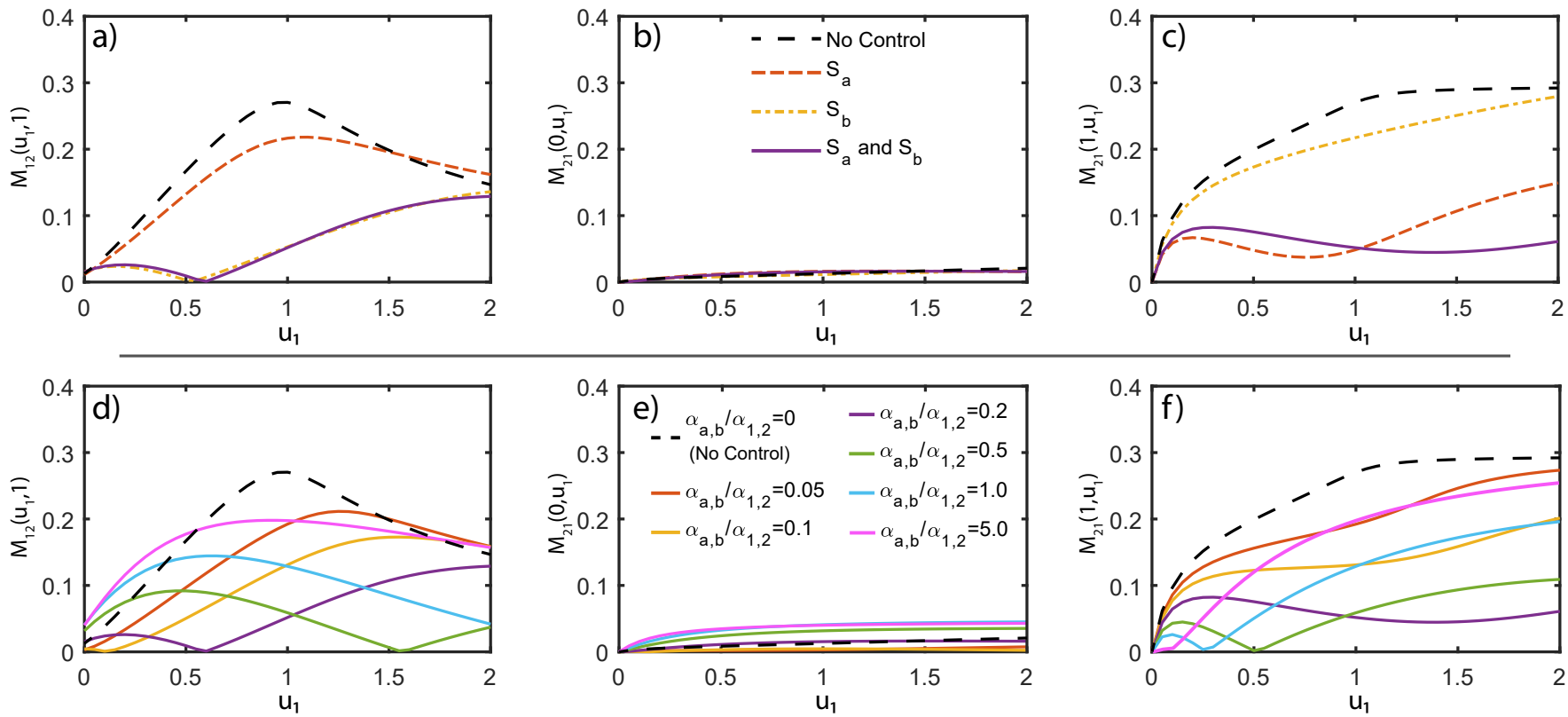

Fig. 4: The proposed control architecture is able to reduce significant cross-talk. a,b,c) Mitigation of cross-talk as parameterised by $M_{i j}$ by the no control (open-loop), one sided, and two-sided control schemes. a) demonstrates the degree of cross-talk observed in $S_{1}$ if $u_{2}$ is changed from 0 to 1 for various $u_{1}$ values. b) demonstrates the degree of cross-talk observed in $S_{1}$ when $u_{2}=0$ and $u_{1}$ varies. c) is similar to $\mathbf{b}$, but with $u_{2}=1$. Values of $S_{i}^{P} / S_{1}^{T}$ for these simulations are presented in Fig. S1. d,e,f) similar calculations to a,b,c for the two-sided control scheme with varying values of feedback parameters $\alpha_{a, b}$. We observe that $M_{12}$ and $M_{21}$ are reduced across most input combinations when $\alpha_{a, b} \in[0.1,1.0]$, though additional cross-talk can be introduced by the control system particularly when $\alpha_{a, b}$ is large (e.g. in panel d when $u_{1}$ is small). Values of $S_{i}^{P} / S_{1}^{T}$ for these simulations are presented in Fig. S2.

use the results developed in [25]. We consider two closed-loop architectures: i) $v_{1}=\alpha_{b} S_{2}^{P}, v_{2}=0$, and ii) $v_{2}=\alpha_{a} S_{1}^{P}$, $v_{1}=0$, finding that their steady-states are attractive for the nominal parameter values, $u_{1}=u_{2}=1$, and different feedback strengths $\alpha_{a}, \alpha_{b}$. This analysis can be applied for all nonnegative parameter and input values. We also comment on the case where both feedback signals $v_{1}, v_{2}$ are active, but we note that analysis in this case becomes more involved.

\section{DISCUSSION}

The proposed feedback control scheme demonstrates a good ability to improve orthogonality of synthetic TCSS, ensuring that the fraction of each substrate in its phosphorylated state is determined primarily by its paired Histidine Kinase's signal level. The two-sided scheme (purple in Fig. 4a-c) achieves this over a wide range of inducer inputs, and we provide an algorithm for assessing its stability for particular parameter values. Implementation of this particular control scheme requires construction of "sink" substrates $S_{a}$ and $S_{b}$, for which interactions with each Histidine Kinase are similar to those of $S_{2}$ and $S_{1}$ respectively. For signals to be transmitted reliably to any interconnected systems we also desire that the $S_{a, b}^{P}$ do not have the same catalytic activity as $S_{2,1}^{P}$ (i.e. they do not act as transcription factors for promoters recognised by $S_{2,1}^{P}$ ). Proteins $S_{a, b}$ with such functionality could be achieved experimentally by mutating the DNA binding domains of $S_{2,1}$, whilst maintaining them as a target for phosphorylation.
The models employed in this paper provide an intuitive description of TCSS in line with previous studies [18], but drastically simplify the complex biochemical reactions taking place. The impact of factors such as the shared phosphate pool or variable rates of Histidine Kinase phosphorylation (governed by the parameters $c_{1,2}$ ) are not considered. Nor is the sequestration of substrate proteins by downstream processes with which they interact, which can have significant impact upon some synthetic biological circuits [26]. Furthermore, all inter-species interactions (i.e. between substrate and Histidine Kinase) are assumed to occur instantly (without intermediate binding steps), which does not account for additional crosstalk due to enzyme sequestration as can arise in some limiting cases [27]. Where possible we have selected parameters (Table I) that reflect the results of experimental studies, though their values may vary depending on the particular implementation and TCSS chosen [19]. Achieving desired control performance would require tuning of the system's feedback strength and the rates of substrate production relative to their interactions with each Histidine Kinase (See Supplementary Sections II and III). This could be achieved experimentally by adjusting the Ribosome Binding Site strengths that govern translation rates of each component.

Future development of the control systems proposed herein will include further investigation of their parameter sensitivity, as well as the variability of their performance when the govern- 
ing equations are simulated stochastically (which can provide useful insights in the analysis of biological control systems [28]). Alternate control schemes may also be considered, depending upon the biological architecture chosen to implement a particular signalling functionality. Theoretical analysis will provide a base to inform experimental implementation, for which the significant challenges include that posed by the protein engineering required to create control species $S_{a, b}$. Realisation of the orthogonal, controlled synthetic signalling pathways described herein will then provide an important building block for Multiple-Input Multiple-Output (MIMO) control systems in synthetic biology.

\section{CONCLUSION}

In this paper we have examined the crosstalk presented by two parallel Two Component Signalling Systems (TCSS), demonstrating that in an un-controlled system off-target interactions of Histidine Kinase enzymes can result in interdependence of signalling state in each pathway. We have proposed and analysed a feedback control architecture to reduce the influence of this crosstalk, demonstrating that it can (stably) provide orthogonality for signals passed through each pathway. Further development and experimental realisation of such control systems will provide valuable capabilities as increasingly complex synthetic biological signalling networks are developed in the future.

\section{REFERENCES}

[1] M. Perego, "Kinase-phosphatase competition regulates Bacillus subtilis development," Trends in Microbiology, vol. 6, no. 9, pp. 366-370, 1998.

[2] M. T. Laub and M. Goulian, "Specificity in Two-Component Signal Transduction Pathways," Annu. Rev. Genet., no. 41, pp. 121-145, 2007.

[3] N. E. Buchler and F. R. Cross, "Protein sequestration generates a flexible ultrasensitive response in a genetic network," Molecular Systems Biology, vol. 5, no. 272, pp. 1-7, 2009.

[4] E. C. OShaughnessy, S. Palani, J. J. Collins, and C. A. Sarkar, "Tunable signal processing in synthetic MAP kinase cascades," Cell, vol. 144, no. 1, pp. 119-131, 2011.

[5] B. W. Andrews, T. M. Yi, and P. A. Iglesias, "Optimal noise filtering in the chemotactic response of Escherichia coli," PLoS Computational Biology, vol. 2, no. 11, pp. 1407-1418, 2006.

[6] V. Chickarmane, B. N. Kholodenko, and H. M. Sauro, "Oscillatory dynamics arising from competitive inhibition and multisite phosphorylation," Journal of Theoretical Biology, vol. 244, no. 1, pp. 68-76, 2007.

[7] J. Hansen, E. Mailand, K. K. Swaminathan, J. Schreiber, B. Angelici, and Y. Benenson, "Transplantation of prokaryotic two-component signaling pathways into mammalian cells," Proceedings of the National Academy of Sciences, vol. 111, no. 44, pp. 15 705-15 710, 2014.

[8] G. Macbeath, "Protein microarrays and proteomics," Nature Genetics, vol. 32, no. 4S, pp. 526-532, 2002.

[9] D. P. Brazil and B. A. Hemmings, "Ten years of protein kinase B signalling: A hard Akt to follow," Trends in Biochemical Sciences, vol. 26 , no. 11 , pp. 657-664, 2001.

[10] D. Danielpour and K. Song, "Cross-talk between IGF-I and TGF- $\beta$ signaling pathways," Cytokine and Growth Factor Reviews, vol. 17, no. $1-2$, pp. $59-74,2006$

[11] M. R. Junttila, S.-P. Li, and J. Westermarck, "Phosphatase-mediated crosstalk between MAPK signaling pathways in the regulation of cell survival," The FASEB Journal, vol. 22, no. 4, pp. 954-965, 2007.

[12] B. Ghosh, U. Sarma, V. Sourjik, and S. Legewie, "Sharing of Phosphatases Promotes Response Plasticity in Phosphorylation Cascades," Biophysical Journal, vol. 114, no. 1, pp. 223-236, 2018.

[13] S. M. Hill, "Receptor crosstalk: Communication through cell signaling pathways," Anatomical Record, vol. 253, no. 2, pp. 42-48, 1998.
[14] B. J. Mayer, M. L. Blinov, and L. M. Loew, "Molecular machines or pleiomorphic ensembles: Signaling complexes revisited," Journal of Biology, vol. 8, no. 9, 2009.

[15] M. Amin, V. B. Kothamachu, E. Feliu, B. E. Scharf, S. L. Porter, and O. S. Soyer, "Phosphate Sink Containing Two-Component Signaling Systems as Tunable Threshold Devices," PLoS Computational Biology, vol. 10, no. 10, 2014.

[16] T. Jin and M. Inouye, "Ligand Binding to the Receptor Domain Regulates the Ratio of Kinase to Phosphatase Activities of the Signaling Domain of the Hybrid Escherichia coli Transmembrane Receptor, Taz1," Journal of Molecular Biology, vol. 232, pp. 484-492, 1993.

[17] K. M. Michalodimitrakis, V. Sourjik, and L. Serrano, "Plasticity in amino acid sensing of the chimeric receptor Taz," Molecular Microbiology, vol. 58 , no. 1 , pp. 257-266, 2005.

[18] E. S. Groban, E. J. Clarke, H. M. Salis, S. M. Miller, and C. A. Voigt, "Kinetic Buffering of Cross Talk between Bacterial Two-Component Sensors," Journal of Molecular Biology, vol. 390, no. 3, pp. 380-393, 2009.

[19] K. Yamamoto, K. Hirao, T. Oshima, H. Aiba, R. Utsumi, and A. Ishihama, "Functional characterization in vitro of all two-component signal transduction systems from Escherichia coli," Journal of Biological Chemistry, vol. 280, no. 2, pp. 1448-1456, 2005.

[20] M. A. Rowland and E. J. Deeds, "Crosstalk and the evolution of specificity in two-component signaling," Proceedings of the National Academy of Sciences, vol. 111, no. 15, pp. 5550-5555, 2014.

[21] T. Yoshida, S. J. Cai, and M. Inouye, "Interaction of EnvZ, a sensory histidine kinase, with phosphorylated OmpR, the cognate response regulator," Molecular Microbiology, vol. 46, no. 5, pp. 1283-1294, 2002.

[22] C. E. Grimshaw, S. Huang, C. G. Hanstein, M. A. Strauch, D. Burbulys, L. Wang, J. A. Hoch, and J. M. Whiteley, "Synergistic kinetic interactions between components of the phosphorelay controlling sporulation in Bacillus subtilis," Biochemistry, vol. 37, no. 5, pp. 1365-1375, 1998.

[23] S. J. Cai and M. Inouye, "EnvZ-OmpR interaction and osmoregulation in Escherichia coli," Journal of Biological Chemistry, vol. 277, no. 27, pp. $24155-24161,2002$.

[24] D. Angeli and E. D. Sontag, "Monotone Control Systems," vol. 48, no. 10 , pp. $1684-1698,2003$.

[25] D. Angeli, G. A. Enciso, and E. D. Sontag, "A small-gain result for orthant-monotone systems under mixed feedback," Systems and Control Letters, vol. 68, no. 1, pp. 9-19, 2014.

[26] D. Mishra, P. M. Rivera, A. Lin, D. Del Vecchio, and R. Weiss, "A load driver device for engineering modularity in biological networks," Nature Biotechnology, vol. 32, no. 12, pp. 1268-1275, 2014.

[27] M. A. Rowland, W. Fontana, and E. J. Deeds, "Crosstalk and Competition in Signaling Networks," Biophys, vol. 103, no. 11, pp. 2389-2398, 2012.

[28] C. Briat, A. Gupta, and M. Khammash, "Antithetic Integral Feedback Ensures Robust Perfect Adaptation in Noisy Biomolecular Networks," Cell Systems, vol. 2, no. 1, pp. 15-26, 2016. 